\title{
Formation and Trapping of Benzyne
}

Burra Shashidher $^{1 *}$, Ramu Bajjuri² and Vijaykumar Guguloath ${ }^{3}$

${ }^{1}$ St.Peter's College of Pharmacy, Madikonda, Warangal, Andhrapradesh, India-506 142

${ }^{2}$ Kingston University, Penrhyn Road, Kingston KT1 2EE, United Kingdom

3 Janagoan Institute of Pharmaceutical Sciences, Jangoan, Warangal, India

\begin{abstract}
Over the decades, several scientists have given a best representation for the structure of benzene. In this present study, Formation of benzyne and trapping achieving with by using furan and tetraphenylcyclopentadienone. The melting point of the crystalline compound was determined and the compound was analysed by InfraRed (IR) , GasChromatography-Mass Spectroscopy(GC-MS), NMR $\left({ }^{1} \mathrm{H}\right)$. IR confirmed that peak, might be due to carbonyl group present in the compound. According FTIR spectrum, strong and broad peaks were predicting may be due to atmospheric carbon dioxide and clearly showing aromatic C-C at $1688.6 \mathrm{~cm}^{-1}$ and $1597.1 \mathrm{~cm}^{-1}$. GC-MS spectra results proving, all the compounds molecular weight is almost nearer to reference spectra. NMR spectra can explaining the structure of the compound by showing chemical shift of functional groups and correlating with the reference. Finally, concluded formation and trapping of benzyne producing adducts such as 1,4-dihydronaphthalene-1,4-endoxide and 1, 2, 3, 4-tetraphenylnaphthalene compounds identifying in dark brown colour and crystalline in nature. The obtained solid products results are compared with that of the literature showing there is no mean deviation in their melting points.
\end{abstract}

Keywords: Benzyne; Trapping; Furan; Chromatography

\section{Introduction}

Over the decades, several scientists have given a best representation for the structure of benzene, that is indeed hexagonal with each C-C bond length being identical $(140 \mathrm{pm})$ and intermediate distance was explained by electron (p-orbital from each neighbouring $\mathrm{C}$ overlap) which extends around the ring giving added stability and decreased reactivity [1].

The compound $\mathrm{C}_{6} \mathrm{H}_{4}$, which is referred as 'Benzyne', is formally derived from benzene by removal of a pair of adjacent hydrogen atoms and the formation of a triple bond.

\section{Chemistry of arynes}

In chemistry, an aryne is an uncharged reactive intermediate derived from an benzene by removal of two ortho substituents leaving two orbitals with two electrons distributed between them. Benzyne is a general class of reactive intermediates known as 2 arynes.

Arynes can undergo three types of mechanisms:

- Nucleophilic aromatic substitution

- Cyclo addition reactions

Nucleophilic aromatic substitution: Nucleophilic aromatic substitution is an addition-elimination reaction that requires at least one substituent which is strongly electron-withdrawing by resonance to stabilise the anionic addition intermediate.

Some reactions explained by elevating a benzyne intermediate: In the below reaction chlorobenzene, having an isotopic labelled carbon atom gives substitution product (aniline-labelled carbon jumbled between two isotopes) and the reaction proceeds through a symmetrical intermediate benzyne, in which two anilines were equivalent $[2,3]$

Cycloaddition reactions: Cycloaddition reactions are those in which two or more unsaturated molecules combine with the formation of a cyclic adduct.
In the below example aryne intermediates are generated and then undergoes further reaction.

For many synthetic applications of arynes it is necessary to generate the reactable intermediate under mild conditions. These behave as intermediates in many organic syntheses.

Applications of arynes:

Synthesis of an Aporphine alkaloid

Synthesis of Ellipticene, an anti-cancer alkaloid

Synthesis of a Lysergic Acid N,N-Diethylamide precursor etc.

\section{Benzyne}

Benzyne is the parent molecule of aryne $(-y n e=$ triple bond) compounds, derivative of benzene, produced by abstraction of two hydrogen atoms especially one produced by abstraction from neighbouring atoms to form a formal triple bond. Benzyne is a reactive intermediate in some reactions involving benzene compounds. More specifically, it is often found as a result of an elimination reaction with substituted aromatic compounds like halogenated or acylated benzene rings. The lifetime of benzyne (gas phase) is 20 nanoseconds $\left(2 \times 10^{-8}\right.$ seconds), Benzyne is very reactive and rapidly dimerise.

Elimination of the substituent, often by nucleophilic attack, results in a carbocation. This electronic arrangement, due to its instability,

*Corresponding author: Burra Shashidher, St.Peter's College of Pharmacy, Madikonda, Warangal, Andhrapradesh, India-506 009, Tel: +919885886988, E-mail: shashidher85@gmail.com

Received July 15, 2011; Accepted September 19, 2011; Published September 21, 2011

Citation: Shashidher B, Bajjuri R, Guguloath V (2011) Formation and Trapping of Benzyne. Pharm Anal Acta 2:137. doi:10.4172/2153-2435.1000137

Copyright: (c) 2011 Shashidher B, et al. This is an open-access article distributed under the terms of the Creative Commons Attribution License, which permits unrestricted use, distribution, and reproduction in any medium, provided the original author and source are credited. 
<smiles>c1ccccc1</smiles>

Figure 1: Structure of benzene.<smiles>Nc1ccccc1NCNc1cccc(Cl)c1</smiles>

$50 \%$<smiles>Nc1ccccc1</smiles>

$50 \%$

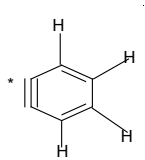

BENZYNE
CHLOROBENZENE

Figure 2: Formation of benzyne from Chlorobenzene.

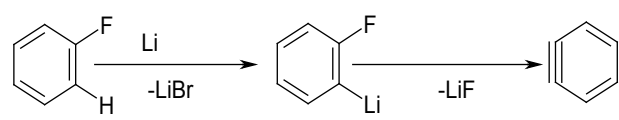

FLUOROBENZENE

BENZYNE

Figure 3: Formation of benzyne from Fluorobenzene.
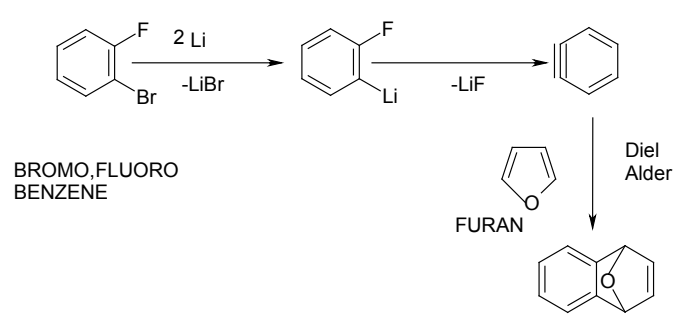

DIHYDRO-EPOXYNAPHTHALENE

Figure 4: Formation of benzyne from halosubstutedbenzene.

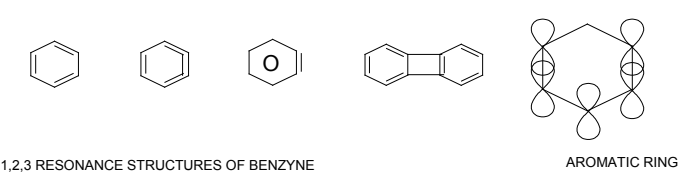

Figure 5: Structure of Benzyne.

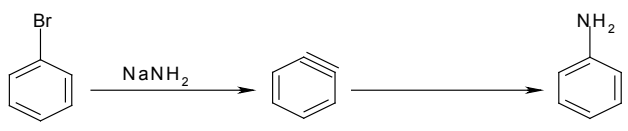

BROMOBENZENE BENZYNE ANILINE

Figure 6: Formation of benzyne from Bromobenzene.

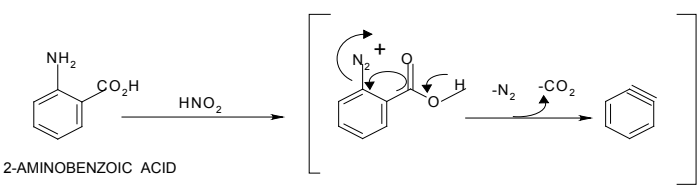

Figure 7: Formation of benzyne intermediate.

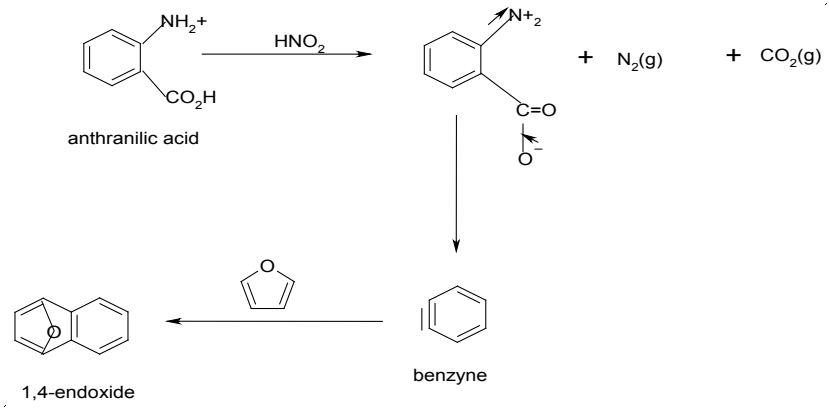

Figure 8: Decomposition of benzyne.

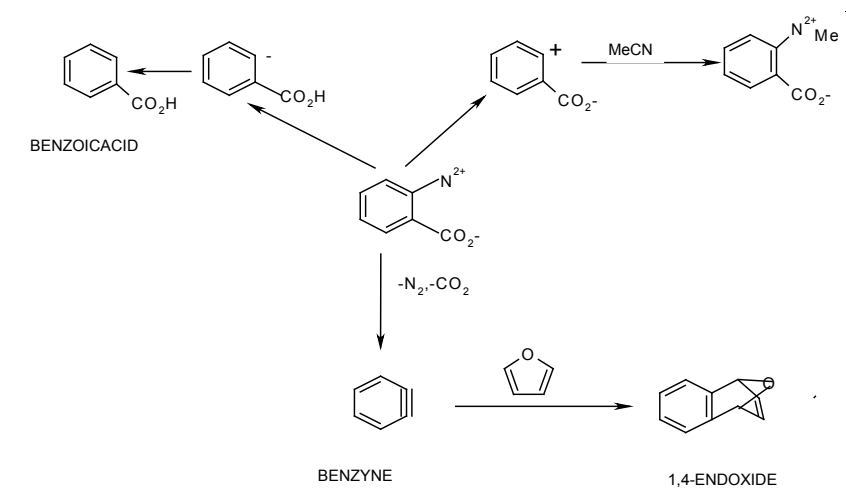

Figure 9: Formation of benzyne.

\begin{tabular}{|l|l|l|}
\hline \multirow{2}{*}{ Products } & \multicolumn{2}{|l|}{ Melting points range (in $\left.{ }^{\circ} \mathrm{C}\right)$} \\
\cline { 2 - 3 } & Experiment & Literature \\
\hline 1,4-dihydro-1,4-epoxynaphthalene & $53.5-55.5^{\circ} \mathrm{C}$ & $54-57^{\circ} \mathrm{C}$ \\
\hline 1-naphthalenol & $94-95^{\circ} \mathrm{C}$ & $94-96^{\circ} \mathrm{C}$ \\
\hline $1,2,3,4$-tetraphenylnaphthalene & $200-201^{\circ} \mathrm{C}$ & $202-204^{\circ} \mathrm{C}$ \\
\hline
\end{tabular}

Table 1: Explain about the products melting point range.

causes spontaneous (or base-assisted in the case of hydrogen) elimination of adjacent substituents resulting in a benzene ring with a triple bond.

In the above mechanism benzyne can be derived from halobenzene but this reaction needs a strong base such as $\mathrm{NaNH}_{2}, \mathrm{BuLi}$. This reagent undergoes elimination reaction to form benzyne intermediate which traps nucleophilic species.

Another possible mechanism is the abstraction of a group such as hydrogen resulting in a carbanion which causes the elimination of an adjacent group. Both mechanisms result in the formation of a benzyne intermediate.

In the above reaction diazotization of 2-aminobenzoic acid was carried out by using nitrous acid resulting a diazonium salt, which is not isolated, but allowed to undergo loss of $\mathrm{CO}_{2}$ and $\mathrm{N}_{2}$ to generate benzyne [4-6].

Benzyne formation and the stepwise decomposition of benzenediazonium-2-carboxylate and trapping

Common usage of the benzyne product involves a Diels-Alder Cyclo addition reaction. Reacting a diene with benzyne taking the place of normal alkene resulting in a bicyclic compound, one ring being 


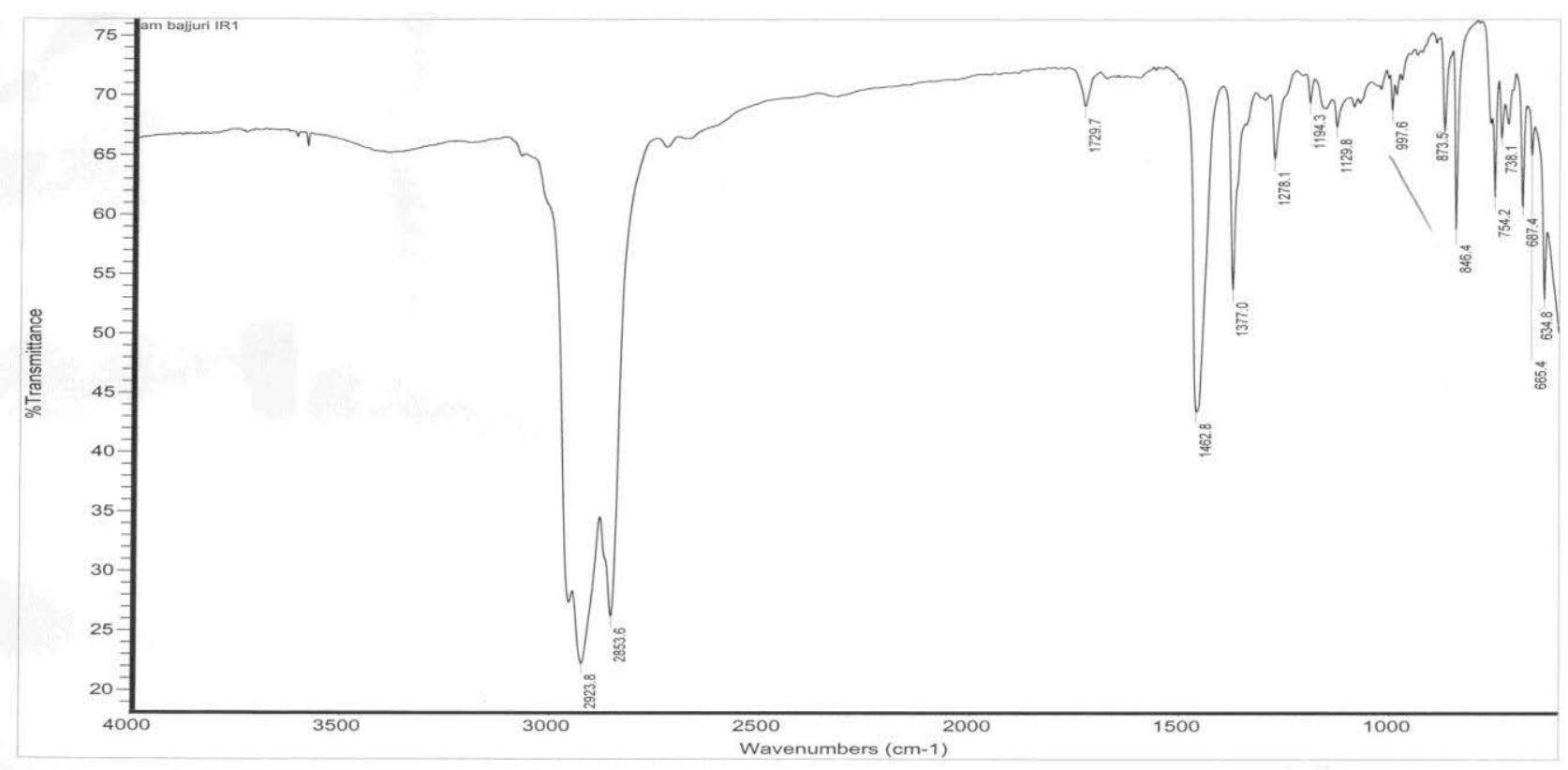

Figure 10: IR Spectra of 1,4-dihydronaphthalene-1,4-endoxide.

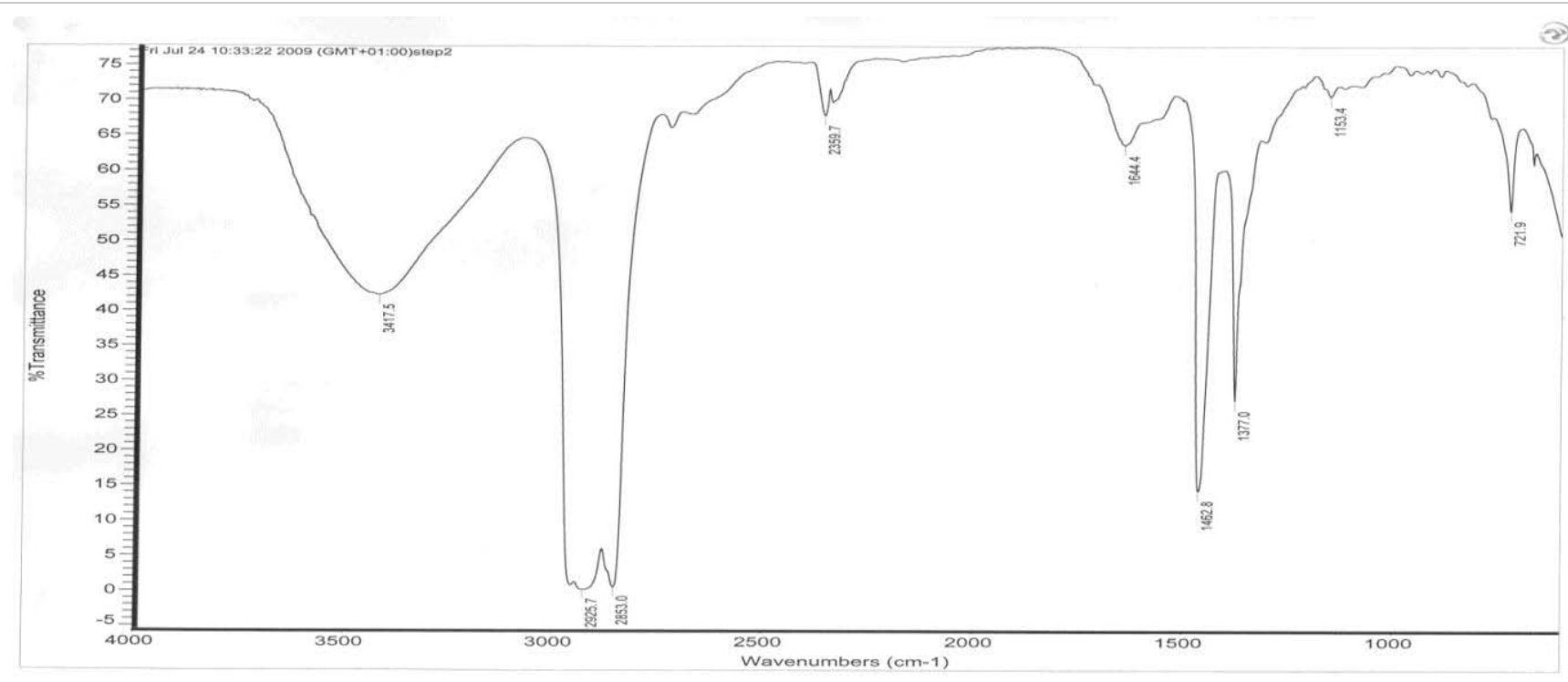

Figure 11: IR Spectra of 1-Naphthalenol.

benzene, the other being the product of the Diels-Alder reaction.

Diazotisation of anthranilic acid is a common method to produce benzyne and substituted benzynes after the loss of nitrogen and carbon dioxide from the neutral diazonium salt. Benzenediazonium2 -carboxylate may be generated in situ from anthranilic acid by diazotisation in an aprotic medium, or by the removal of the elements of hydrogen chloride from 2-carboxybenzenediazoniumchloride. Benzenediazonium-2-carboxylate is a violently explosive benzyne precursor [7-9].

Benzyne formation is favoured in halogenated solvents and occurs by the concentrated loss of nitrogen and carbon dioxide.

\section{Experimental}

\section{Materails}

Anthranilic acid $\left(2-\left(\mathrm{H}_{2} \mathrm{~N}\right) \mathrm{C}_{6} \mathrm{H}_{4} \mathrm{CO}_{2} \mathrm{H}\right)$, 1,2-dimethoxyethane $\left(\mathrm{CH}_{3} \mathrm{OCH}_{2} \mathrm{CH}_{2} \mathrm{OCH}_{3}\right.$, Tetraphenylcyclopentadienone and Sodium hydroxide are obtained from Sigma- Aldrich, United Kingdom.; Isoamyl nitrite, Furan $\left(\mathrm{C}_{4} \mathrm{H}_{4} \mathrm{O}\right)$ and Petroleum ether samples obtained from Fluka, United kingdom.

\section{Methods}

Two methods were carried out to derive the benzyne. The methods are as follows: 


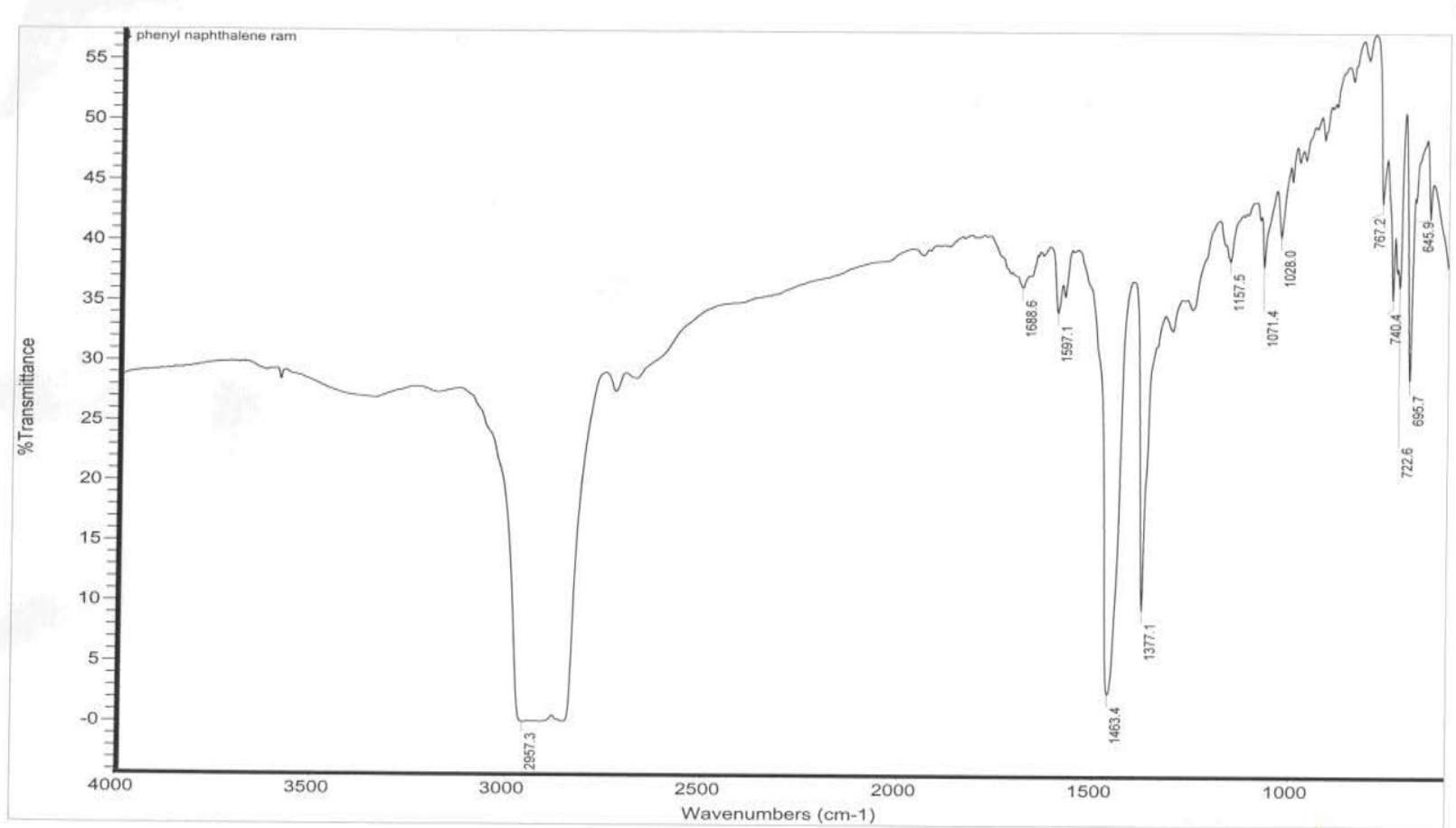

Figure 12: IR Spectra of 1,2,3,4-tetraphenylnaphthalene.

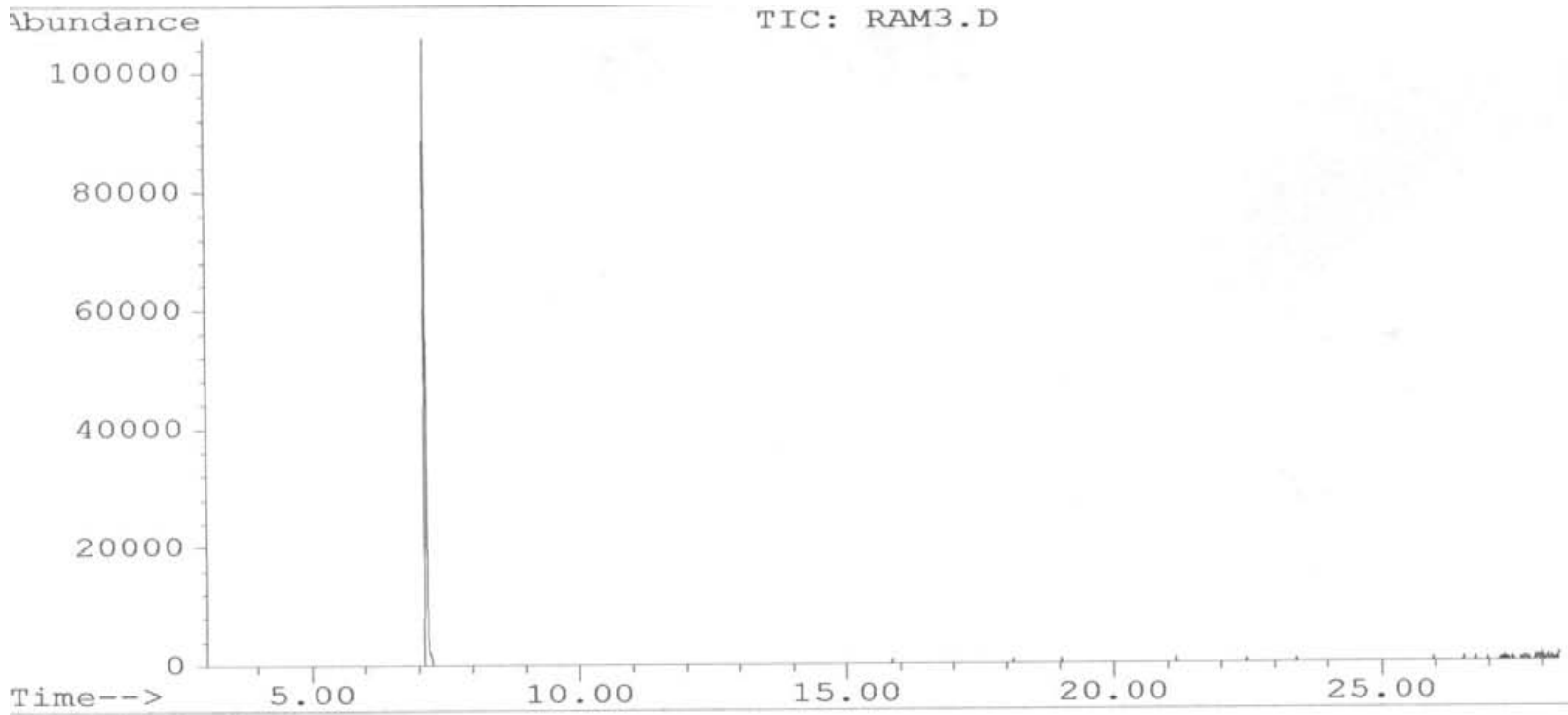

Figure 13: GC-MS Spectra of 1,4-dihydronaphthalene-1,4-endoxide.

1. Formation of benzyne and trapping with furan.

2. Formation of benzyne and trapping with tetraphenylcyclopentadienone.

Formation of benzyne and trapping with furan: $10 \mathrm{ml}$ of Furan (Mol.Wt 68.07) and $10 \mathrm{ml}$ of 1, 2-dimethoxyethane (Mol. Wt 90.12) were taken in a $100 \mathrm{ml}$ round bottomed flask, some bumping stones were added. The round bottomed flask was fitted to a reflux condenser and the solution was heated under steam. Mean while, two solutions were prepared in $25 \mathrm{ml}$ Erlenmeyer flasks first one containing, $4 \mathrm{ml}$ of iso-amyl nitrite made up to $10 \mathrm{ml}$ with 1,2-dimethoxyethane and second solution containing $2.74 \mathrm{gm}$ anthranilic acid with 1,2-dimethoxyethane. $2 \mathrm{ml}$ of each solution was added by using separate Pasteur pipettes through the condenser to the round bottom flask under steam with 8-10 min intervals. The solution turned to a dark orange colour after the solutions were fully added. Then the mixture 


\begin{tabular}{|l|l|}
\hline Fragment & mass-to-charge ratio $(\mathrm{m} / \mathbf{z})$ \\
\hline $\mathrm{C}_{10} \mathrm{H}_{8}\left(\mathrm{M}^{+}\right)$ & 144 \\
\hline $\mathrm{C}_{10} \mathrm{H}_{8}^{+}$ & 128 \\
\hline $\mathrm{C}_{8} \mathrm{H}_{6} \mathrm{O}^{+}$ & 118 \\
\hline $\mathrm{C}_{9} \mathrm{H}_{7}^{+}$ & 115 \\
\hline $\mathrm{C}_{7} \mathrm{H}_{5}^{+}$ & 89 \\
\hline
\end{tabular}

Table 2: Interpretation of GC-MS results Of 1,4-dihydro-1,4-epoxynaphthalene. mixture was washed with $15 \mathrm{ml}$ of distilled water for 2 times. Then the organic solution was dried over magnesium sulphate (drying agent), the drying agent was removed by suction. Finally the solution was decolourized with active charcoal powder. Then the colourless solution was put into rotatory-evaporator to obtain crystalline slurry by applying room temperature and pressure. Crystalline slurry obtained was repurified by sublimation using ice cold petroleum ether [10].

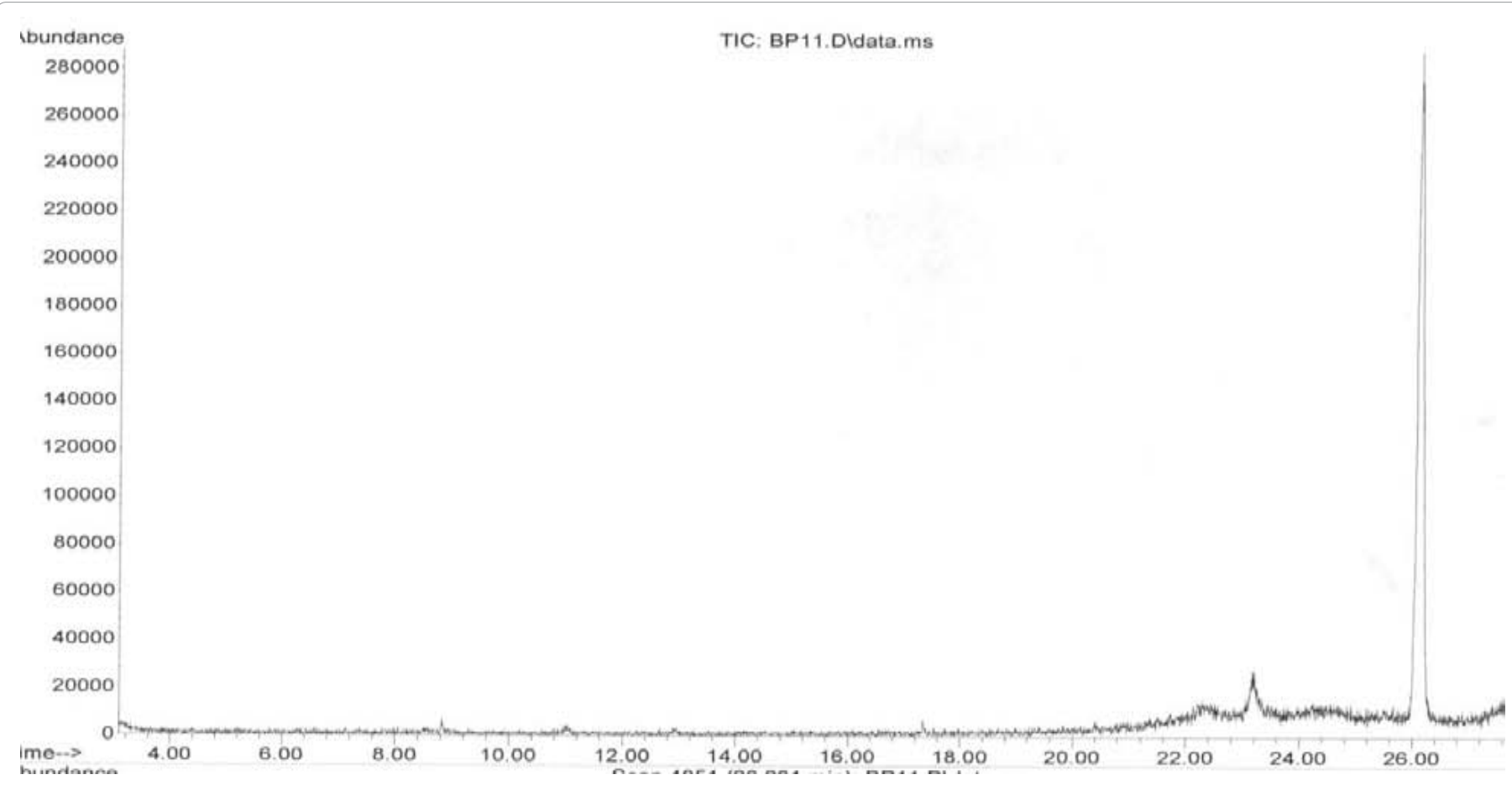

Figure 14: GC-MS Spectra of 1,2,3,4-Tetraphenylnaphthalene.

was refluxed for $30 \mathrm{~min}$, on cooling this mixture to room temperature a dark brown mixture was precipitated out.

In the mean time $\mathrm{NaOH}(0.1 \mathrm{M})$ solution was prepared and added to dark brown mixture. The mixture was then transferred to $100 \mathrm{ml}$ separating funnel, organic layer was separated by extracting the product with $15 \mathrm{ml}$ petroleum ether. This procedure was repeated for three times to get efficient organic solution. The organic mixture was washed with $15 \mathrm{ml}$ of distilled water for 6 times. Then the organic solution was dried over magnesium sulphate (drying agent) and it was removed by suction. Finally the solution was decolourized with active charcoal powder, the solution was taken into rotatory-evaporator to obtain colour less crystalline slurry by applying room temperature and pressure. Crystalline slurry obtained was repurified by sublimation using ice cold petroleum ether [10].

Treatment of 1, 4-dihydronaphthalene-1, 4-endoxide with acid: This step is optional for the method development.1,4dihydronaphthalene-1,4-endoxide (432 gm) was placed in $25 \mathrm{ml}$ Erlenmeyer flask and dissolved in ethanol (10 ml), concentrated $\mathrm{HCl}(5$ $\mathrm{ml}$ ) was added to the solution and placed at room temperature for one hour. After one hour the mixture was poured into $100 \mathrm{ml}$ separating funnel, by adding diethyl ether $(20 \mathrm{ml})$. Extraction of the organic layer was carried out by shaking the separating funnel. Then the organic
Formation of benzene and trapping with tetracyclopentadienone: Both Tetraphenylcyclopentadienone $(0.3 \mathrm{M}), 1$, 2-dimethoxyethane (DME) were placed in $100 \mathrm{ml}$ round bottom flask fitted with an efficient reflux condenser and the solution was heated further under steam. On the other hand, two solutions were prepared in $25 \mathrm{ml}$ Erlenmeyer flasks first one containing iso-amyl nitrite $(4 \mathrm{ml})$ made up to $10 \mathrm{ml}$ with 1,2-dimethoxyethane and second solution containing $2.74 \mathrm{gm}$ of anthranilic acid with 1,2-dimethoxyethane. $2 \mathrm{ml}$ of each solution were added by using separate Pasteur pipettes through the condenser to the round bottom flask under steam with 8-10 min intervals. The solution turned in to a dark orange colour after the solutions were fully added. Then the mixture was refluxed for $30 \mathrm{~min}$, on cooling the mixture to room temperature a dark brown mixture was precipitated out.

In the Mean time $\mathrm{NaOH}(0.1 \mathrm{M})$ solution was prepared and added to dark brown mixture. And the mixture was transferred to 100 $\mathrm{ml}$ separating funnel, organic layer was separated by extracting the product with petroleum ether $(15 \mathrm{ml})$. This procedure was repeated for three times to get efficient organic solution. Again the organic mixture was washed with $6 \times 15 \mathrm{ml}$ distilled water. Then the organic solution was dried over magnesium sulphate (drying agent) and it is removed by suction. Finally the solution was decolourized with active charcoal powder. Then the colourless solution was put into rotatory-evaporator to obtain yellow crystalline slurry by applying room temperature and 


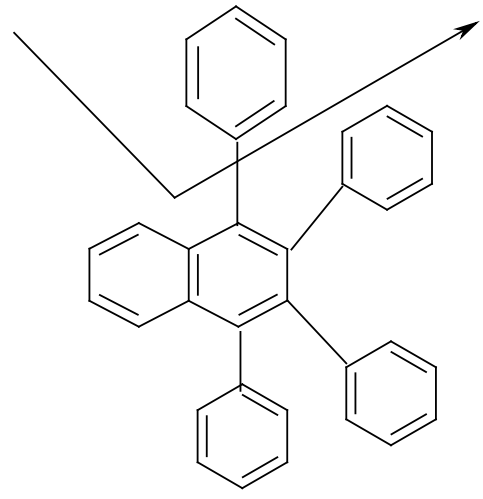

$355 \& 276$

Figure 15: Fragmentation of 1,2,3,4-Tetraphenylnaphthalene.

\begin{tabular}{|l|l|}
\hline Fragment & mass-to-charge ratio(m/z) \\
\hline $\mathrm{C}_{34} \mathrm{H}_{24}\left(\mathrm{M}^{+}\right)$ & 432 \\
\hline $\mathrm{C}_{28} \mathrm{H}_{19}{ }^{+}$ & 355 \\
\hline $\mathrm{C}_{12} \mathrm{H}_{24}{ }^{+}$ & 156 \\
\hline $\mathrm{C}_{6} \mathrm{H}_{5}^{+}$ & 77 \\
\hline
\end{tabular}

Table 3: Interpretation of GC-MS results of the 1,2,3,4-Tetraphenylnaphthalene.

pressure. Crystalline slurry obtained was repurified by sublimation using ice cold petroleum ether.

The melting point of the crystalline compound was determined and the compound was analysed by InfraRed (Nujol), GasChromatographyMass Spectroscopy, NMR $\left({ }^{1} \mathrm{H}\right)[10]$.

\section{Instrumentation}

Infrared spectroscopy: Infrared spectroscopy is used in the determination of functional groups in organic materials, determination of the molecular composition of surfaces, Identification of chromatographic effluents, Determination of molecular conformation (structural isomers) and stereochemistry

And determination of molecular orientation. FT-IR spectra were recorded on a PerkinElmer Paragon 1000 FT-IR and Processing software, Copyright (c) 2005 PerkinElmer Instruments LLC. It is used for the identification and structural analysis of organic compounds in that absorption of electromagnetic radiation occurs due to changes in the vibrational energy of the molecules, It influences on molecular vibrations and rotational transitions. Substance was crushed in a mortar to make a fine powder and mixed further with nujol to make a slurry. It was rubbed on glass disc and placed in a chamber for analysis [11].

\section{Gas Chromatography-Mass Spectroscopy (GC-MS)}

GC-MS is a method that combines the features of gas-liquid chromatography and mass spectrometry to identify and quantitation of volatile and semivolatile organic compounds in complex mixtures within a test sample, determination of molecular weights, Structural determination of unknown organic compounds in complex mixtures both by matching their spectra with reference spectra and by a priori spectral interpretation $.2 \mathrm{mg}$ of substance was dissolved in $10 \mathrm{ml}$ of light petroleum and filled in a labelled vial and placed in the instrument for the analysis. Mass spectra were recorded using a varian CP-3800 Gas Chromatography with Varian 1200L Quadrupole mass spectrometer [12].

\section{Nuclear Magnetic Resonance Spectrometry (NMR)}

NMR is a technique using for identification and structural analysis of organic compounds and chemical properties of the molecules in which they are contained. The principle behind NMR is that many nuclei have spin and all nuclei are electrically charged. If an external magnetic field is applied, an energy transfer is possible between the base energy to a higher energy level. The energy transfer takes place at a wavelength that corresponds to radio frequencies and when the spin returns to its base level, energy is emitted at the same frequency. The signal that matches this transfer is measured in many ways and processed in order to yield an NMR spectrum for the nucleus concerned. $3 \mathrm{mg}$ of substance was taken in a vial and dissolved it in $\mathrm{CDCl}_{3}$ using sonicator. $1 \%$ of Tetra Methyl Silane (TMS) was added and made the volume up to $5 \mathrm{~cm}$ in a nmr tube. All NMR spectra were recorded at $400 \mathrm{MHz}\left({ }^{1} \mathrm{H}\right)$ on a Jeol Eclipse+ 400 NMR spectrometer using Jeol Delta version 4.3.6 control and processing software, Jeol, USA, Inc. Chemical shifts are reported in ppm, downfield from chloroform- $\mathrm{d}\left(\mathrm{CDCl}_{3}\right)$ as an internal reference [13-15].

\section{Results and Discussion}

The obtained solid products from the above three procedures were analysed to know whether the desired compound was formed or not by various analytical techniques. And these results are compared with that of the literature reference.

\section{Melting point}

1,4-dihydronaphthalene-1,4-endoxide and 1,2,3,4-tetraphenyl naphthalene producing the reproducing yield values i.e. $32.4 \%$ and $71.0 \%$ respectively.

\section{IR spectra of the products}

Infrared spectroscopy is a reliable means of identifying different functional groups present on the product by their characteristic vibration frequency and comparing it with the groups present in the starting material.

1,4-dihydronaphthalene-1,4-endoxide: The FT-IR spectrum obtained for the compound showed the peak at $2923.8 \mathrm{~cm}^{-1}$ which was due to Nujol. This was further confirmed from the peaks $1462.8 \mathrm{~cm}^{-1}$ and $1377 \mathrm{~cm}^{-1}$. The peak $1729.7 \mathrm{~cm}^{-1}$ might be due to carbonyl group present in the compound.

There are other peaks obtained which may be due to the presence of impurities or solvent.

\section{1-Naphthalenol}

The FT-IR spectrum of the compound was obtained. The peak at $3417.5 \mathrm{~cm}^{-1}$ clearly showed 'OH' stretch. The peak was strong and broad. The peak at $2359.7 \mathrm{~cm}^{-1}$ may be due to atmospheric carbon dioxide. Prominent peaks were obtained for Nujol at $2925.7 \mathrm{~cm}^{-1}, 2853$ $\mathrm{cm}^{-1}, 1482.8 \mathrm{~cm}^{-1}$ and $1377 \mathrm{~cm}^{-1}$.

1,2,3,4-tetraphenyl naphthalene: According to the FT-IR spectrum of the compound the peaks obtained clearly showed aromatic $\mathrm{C}-\mathrm{C}$ at $1688.6 \mathrm{~cm}^{-1}$ and $1597.1 \mathrm{~cm}^{-1}$. The peaks at $2957.3 \mathrm{~cm}^{-1}, 1453.4$ $\mathrm{cm}^{-1}$ and $1377.1 \mathrm{~cm}^{-1}$ were due of $\mathrm{Nujol}^{11}$.

\section{Gc-Ms spectra of the products}

The gas chromatography-mass spectrometry is a combination of two techniques, where gas chromatography separates different 


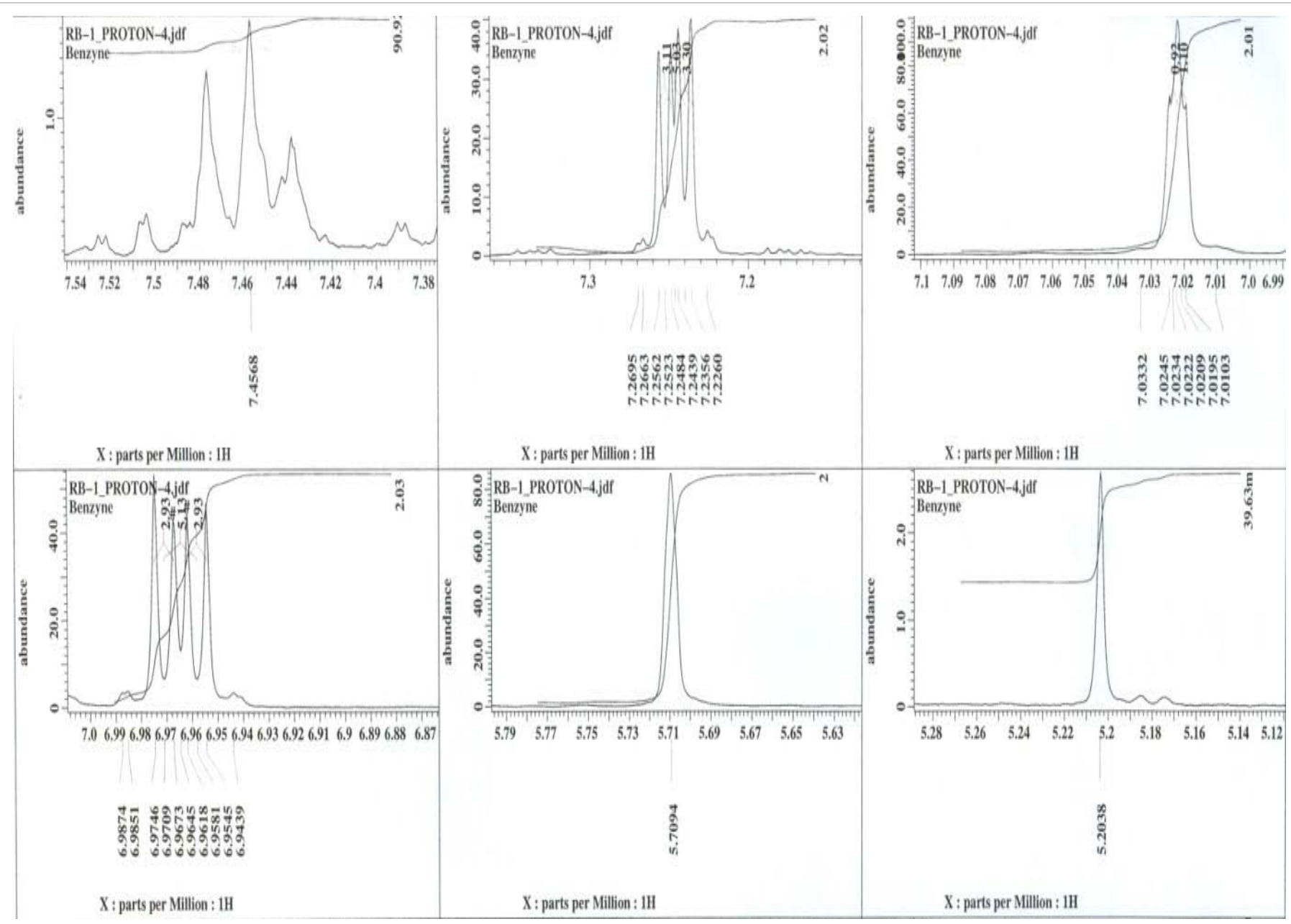

Figure 16: NMR spectrum of 1,4-dihydronaphthalene-1,4-endoxide.

components in the sample and mass spectra provides the structural details by fragmentation of the sample.

\section{1,4-dihydronaphthalene-1,4-endoxide}

The full scan default mode with selected ions at $\mathrm{m} / \mathrm{z} 89,115,118,128$ and 144, was shown in above graph. As it can be seen in the figure, sample was well separated in a run time of 7-7.5 min, and the spectra showing last peak at $144(\mathrm{~m} / \mathrm{z})$.

From the spectra, if we observe the molecular peak at $144 \mathrm{~m} / \mathrm{z}$<smiles></smiles>

Figure 17: Symmetric structure of 1,4-dihydronaphthalene-1,4-endoxide.

\begin{tabular}{|l|l|l|l|}
\hline$\delta(\mathrm{ppm})$ & Multiplicity & Coupling constant J in (hertz) & Integrals \\
\hline 7.26 & Doublet of doublet & $5.03,3.3$ & $2 \mathrm{H}$ \\
\hline 7.0234 & Doublet of doublet & $1.10,0.92$ & $2 \mathrm{H}$ \\
\hline 6.97 & Doublet of doublet & $5.13,2.93$ & $2 \mathrm{H}$ \\
\hline 5.7094 & Doublet of doublet & & $2 \mathrm{H}$ \\
\hline
\end{tabular}

Table 4: Interpretation of NMR results of the 1,4-dihydronaphthalene-1,4-endoxide.

(last peak) and comparing it with the molecular weight of the desired product, and also verifying it with reference library search ensured that this spectra of the compound is almost nearer to reference spectra. Hence I conclude that the product is reliable with the desired product.

1,2,3,4-Tetraphenylnaphthalene: The full scan default mode with selected ions at $\mathrm{m} / \mathrm{z} 77,156,355$ and 432 , was shown in above graph. As it can be seen in the figure, sample was well separated in a run time of 25.5-26.5 min, and the spectra showing most abundant peak at $432(\mathrm{~m} / \mathrm{z})$ following $26 \mathrm{~min}$ retention time. But between $20-26 \mathrm{~min}$ of retention time so many other benzene compounds were eluted which may be due to the reaction mixture or handling procedures.

The fragmentation of 1,2,3,4-tetraphenylnaphthalene, it showed 
loss of phenyl rings from the attached naphthalene ring, simultaneously spectra showing peaks at $\mathrm{m} / \mathrm{z} 355,156,77$ respectively.

From the spectra, if we observe the molecular peak at $432 \mathrm{~m} / \mathrm{z}$, comparing it with the molecular weight of the desired product, and also verifying it with reference library search ensured that this spectra of the compound is almost nearer to reference spectra. Hence I conclude that the product is reliable with the desired product.

Nuclear magnetic resonance -proton spectroscopy: The ' $H$ NMR spectra was obtained by using $\mathrm{CHCl}_{3}$ as solvent and spectra can explain the structure of the compound by showing chemical shift of functional groups.

From the above structure of 1,4-dihydro-1,4-epoxynaphthalene contains symmetrical structure and showing four different types of doublet protons which are indicated as $\mathrm{Ha}, \mathrm{Ha}, \mathrm{Hb}, \mathrm{Hb}, \mathrm{Hc}, \mathrm{Hc}, \mathrm{Hd}$, $\mathrm{Hd}$, and having equal integration ratio, The spectra of 1,4-dihydro1,4-epoxynaphthalene showing that all the four protons are attached to the epoxy naphthalene base and located in same environment and strongly deshielded by the $\pi$ orbitals of the ring and absorbed in the low field, ranging from 5.7094ppm-7.2695ppm. While, the protons $\mathrm{Ha}, \mathrm{Hb}, \mathrm{Hc}$, Hd showing the multiplication at 6.9439-6.9874, 7.2260$7.2695,5.7094,7.0103-7.0332$ respectively. And all these doublets are having one neighbouring protons. $\mathrm{Ha}, \mathrm{Hb}, \mathrm{Hc}, \mathrm{Hd}$ are respective of neighbouring protons $\mathrm{Hb}, \mathrm{Hc}, \mathrm{Hd}, \mathrm{Ha}$ and showed a signal that split into a doublet. Each proton on the structure had two neighbouring protons which indicates one proton is non-equivalent and the other is equivalent and gives a signal that is split into a doublet of doublet.

All the peaks in $\mathrm{Ha}, \mathrm{Hb}, \mathrm{Hc}, \mathrm{Hd}$ mostly similar and remaining peaks were found between $1.219 \mathrm{ppm}-5.2038 \mathrm{ppm}$ which may be due to the impurities or the small changes in the local shielding environment of the $\mathrm{CHCl}_{3}$ induced by the solute via intermolecular interactions.

\section{Conclusion}

Formation and trapping of benzene has been successfully done by using two different trapping agents which are Furan and tetraphenyl cyclopentadienone with significant results.

In the first experiment, benzyne was trapped by furan and the adduct obtained was 1,4-dihydronaphthalene-1,4-endoxide. The yield obtained in the first experiment was low when compare to the second experiment. The compound synthesised was crystalline in nature and yellow orange in colour. The compound was confirmed by its melting point, Infrared, GC-MS and NMR Spectra compared with that of the literature values.

Similarly the benzene was also trapped by using 1, 2, 3, 4-tetraphenylcyclopentadienone. Here, the adduct obtained was 1, 2, 3, 4-tetraphenylnaphthalene. The compound was dark brown in colour and crystalline in nature. It was also confirmed by comparing the obtained melting point values with the literature values.

\section{References}

1. Rocke J (1985) Annals of Science 42.

2. Gilchrist T (2009) Aryne [Online]. Available: Chemistry - Aryne.mht.

3. Moody Whitham (2005) Reactive intermediates. $2^{\text {nd }}$ ed.London: Oxford chemistry primers.

4. Lesterf Riedmaannd Fraxcis, Logullo. (1969) Arynes via Aprotic Diazotization of Anthranilic Acids. J Org Chem 34: 3089-3095.

5. Clark A, Davidson ER (2003) p-Benzyne derivatives that have exceptionally small singlet-triplet gaps and even a triplet ground state. J Org Chem 68: 3387 3396.

6. March P, Sundberg (1967) The Classic account of benzynes. Academy press.

7. Gottfried B, Janet N (1980) The Intra molecular Diels-Alder reaction. Journal of chemical Reviews 80: 63-97.

8. Harwood LM, Moody CJ (2003) Experimental organic chemistry. $2^{\text {nd }} e d$. Oxford: Blackwell science Itd.

9. Wade LG (2002) Formation Benzyne and its Diels Alder reaction: A multi step reaction sequence. CER Modular laboratory program in chemistry 748 .

10. Harwood LM, Moody CJ (2003) Experimental organic chemistry. $3^{\text {nd }} e d$. Oxford: Blackwell science Itd.

11. Lampan $P(2009)$ Introduction to spectroscopy. $4^{\text {th }}$ ed. USA: Blackwell science Itd.

12. Peter G, James A (2007) Fourier Transform Infrared Spectrometry. In Analytical chemistry. $2^{\text {nd }} e d$. Willy Publications.

13. Brimateir E (2002) Structure Elucidation by NMR in Organic Chemistry: A Practical Guide, In Analytical chemistry. Willy Publications.

14. Peter G, James A (2007) Fourier Transform Infrared Spectrometry. In Analytical chemistry. $2^{\text {nd }}$ ed. Willy Publications.

15. Eberhard B (2002) Structure Elucidation by NMR in Organic Chemistry: A Practical Guide, In Analytical chemistry. Willy Publications. 